\title{
TRAYECTO DE UNA INVESTIGACIÓN: EL CASO DE LA COOPERATIVA INTEGRAL CATALANA. DESIGUALDADES, EXCLUSIÓN Y PRECARIZACIÓN
}

\author{
THE PATH OF AN INVESTIGATION: THE CASE \\ OF THE CATALAN INTEGRAL COOPERATIVE. INEQUALITIES, \\ EXCLUSION AND PRECARIOUSNESS
}

\author{
Valeria de Ormaechea Otalora*
}

\begin{abstract}
El presente artículo es producto de una experiencia de investigación sobre un caso particular. El punto de partida fue preguntarse como las transformaciones sociales de los últimos años, aquellas que anteceden y continúan a las movilizaciones del $15 \mathrm{M}^{1}$, han generado nuevos protagonismos sociales y cómo estos han ido configurando espacios de aprendizaje colectivo pensados y practicados desde la horizontalidad. El trayecto de esta investigación nos confrontó con dilemas, contradicciones, responsabilidades y compromisos que supusieron problematizar el rol del/ la investigador/a y la necesidad de asumir un enfoque epistemológico que demanda una relación de igualdad entre los sujetos implicados en dicha investigación. Un trabajo que en esa búsqueda de respuestas se hace investigación-acción y coproducción de saberes y conocimientos situados.

Palabras claves: Investigación participativa, investigación militante, diálogo de saberes, etnografía, nuevos protagonismos sociales.

This article is the product of a research experience from a case study on 15M's social movements. The main reseaarch question consisted in analyzing how the social transformations of the recent years, those that precede and persist after the mobilizations of $15 \mathrm{M}$, have generated new social protagonisms and how these have creted spaces of collective learning conceived and interpreted from an horizontal perspective. The journey of this research confronted us with dilemmas, contradictions, responsibilities and compromises that challenged the researcher's role and the necessity to assume an epistemological approach implying a relation of equality between all subjects involved in the investigation. In this quest for answers, this study merged research and activism and led to the co-sharing of knowledge and situaded knowledge.
\end{abstract}

Key words: Participative research, politically-engaged research, dialog of knowledge, ethnography, new social protagonisms.

\begin{abstract}
De todo, quedaron tres cosas: la certeza de que estaba siempre comenzando, la certeza de que había que seguir y la certeza de que sería interrumpido antes de terminar. Hacer de la interrupción un camino nuevo, hacer de la caída un paso de danza, del miedo una escalera, del sueño un puente, de la búsqueda... un encuentro (Fernando Pessoa, 2008).
\end{abstract}

\section{Introducción}

Este texto relata un recorrido. Un camino que sin querer nos llevo al reconocimiento de algo que no era nuevo, pero que sin embargo nos seguía hablando de la importancia de la experiencia compartida, de que con nuestras prácticas de resistencia, a veces pequeñas y cotidianas, también construimos espacios de saber y conocimiento colectivo que muchas veces van más allá del tema que pretendemos y creemos trabajar. Del recorrido de una investigación que fue etnográfica y que muy pronto, por la necesidad urgente de coherencia y honestidad, se convirtió en lo que provisionalmente llamaré investigación participativa; sabiendo de todo lo que contienen estas dos palabras dejo para un trabajo más extenso profundizar en las derivaciones y amplitud que posee una investigación de este tipo.

La dificultad formal con la que me encontré en la escritura de este texto, el ir y venir de la primera persona del singular a la primera del plural, expresaba que me encontraba batallando con el difícil lugar que asume la investigadora frente a las preguntas de su investigación o mejor al lugar que elige tomar en ella. Dudas que no se agotaban en la decisión metodológica de asumir una investigación

* Universidad Nacional de Educación a Distancia (UNED), España. Correo electrónico: valedeo@ hotmail.com 
de tipo participativa, sino que se ampliaban en una decisión que se hacía acción política al tiempo que investigativa.

El trabajo comenzó en el 2013 y surge de la aproximación y observación de un espacio colectivizado ubicado en el barrio de Sagrada Familia de Barcelona. La investigación se propuso indagar cómo las transformaciones sociales de los últimos años han generado nuevas formas de participación ciudadana y cómo estas, a su vez, han ido configurando verdaderos espacios de aprendizaje colectivo pensados y practicados desde la horizontalidad.

Me interesaba indagar, con una mirada amplia, cuáles eran los procesos, dinámicas, estrategias que se desarrollan al interior de estos espacios y cómo estas generan a su vez zonas de aprendizaje transversal, colaborativo, en muchos casos cercanos a la educación formal, pero en muchos otros replanteando las formas tradicionales de aprender, de generar conocimiento o de participar en la vida comunitaria.

El trabajo se sitúa en un contexto donde las desigualdades, exclusión y precarización de gran parte de la población, no solo en el Estado español sino a nivel global, junto a la crítica y desencanto del modelo democrático actual han generado otras formas de expresión, resistencia y contestación social donde se ensayan diversas maneras de colaboración, relación y apoyo mutuo por medio de los cuales las personas dan respuesta a sus propias demandas. Esto ha dado lugar a la aparición de nuevas filiaciones y formas de construir espacios comunes donde vivir, consumir, producir, decidir, etc., diferentes a los que propone la democracia formal. Aparece entonces un nuevo protagonismo social que se define fundamentalmente por la diversidad de propuestas, discursos y sensibilidades que proponen diversas formas de dar respuestas a la falta de acceso igualitario a bienes materiales y simbólicos.

Podríamos decir que estas nuevas formas de protagonismo apuestan por una construcción social y disputa por el poder impulsados por la autonomía y la autogestión; son más un contrapoder que un nuevo (tradicional) poder estructurado en términos macroinstitucionales (Muñoz Tamayo, 2006). Tamayo nos dice que estas formas organizativas o de asociación se diferencian de los movimientos sociales clásicos, como el movimiento obrero o estudiantil, porque no se definen necesariamente en términos económicoestructurales, sino por el reconocimiento social y la afirmación de la identidad; para el autor se trataría de una definición más cultural que sistémica/económica (Muñoz Tamayo, 2006). En este nuevo escenario no se trata de la "toma del poder", de construir un Estado nuevo, sino de crear y defender experiencias plurales y heterogéneas, de proteger y fomentar otras formas de vida innovadoras (Hardt y Negri, 2002; Virno, 2003; Zibechi, 2007).

\section{El/la investigador/a. Un lugar incómodo}

La investigación se planteó como estudio y profundización de un caso particular que fue el de la Cooperativa Integral Catalana (CIC).

Para la realización de esta investigación confié, en un primer momento, en el acercamiento etnográfico como herramienta de análisis y reflexión, ya que esta es un instrumento fundamental a la hora de abordar y analizar la complejidad de la realidad social por su asertividad y reflexividad. Para Dietz la etnografía no es reducible a un mero instrumento de las ciencias sociales ni a un arma de "liberación" de los "oprimidos" (Díaz de Rada, 2010; Dietz, 2011). La etnografía es un quehacer reflexivo que recupera desde dentro el discurso del actor social, a la vez que desde fuera lo contrasta con su respectiva praxis incorporada (Dietz, 2012).

Esta elección permitió profundizar en los roles, ritos, procesos de institucionalización y modos de participación que se desarrollaban en este espacio. Sin embargo en el momento de la negociación en el acceso al campo surgieron las primeras preguntas de corte ético, estas dudas no fueron insalvables, pero las cuestiones de fondo que planteaban siguieron estando ahí, cambiaron de forma y adquirieron nuevos matices. Estos dilemas hablaban del límite que separa el campo de acción del etnógrafo y el rol de participación y compromiso que puede tener dentro de la comunidad con la que trabaja.

Sabía del difícil lugar que le toca asumir al investigador/ra; ese lugar que no está ni dentro ni fuera, ni lejos ni cerca y que por esto a veces se mueve entre categorías que reclaman ser definidas en nombre del entendimiento, las convenciones académicas o la racionalidad científica.

A medida que avanzaba en el trabajo de campo la experiencia iba abriendo las preguntas. Pensar en un linde resultaba ser un tanto artificioso, pues el investigador/a se mueve en un espacio donde la acción, la participación y el análisis se confunden y se fusionan permanentemente. Sin embargo, al 
revisar la práctica algunas categorías seguían estando ahí, a veces de forma imperceptible, otras de modo más evidente, pero siempre eran una especie de presencia incómoda que me interpelaba desde un lugar donde se abrían tensiones epistémicas al tiempo que éticas.

Es verdad que las lecturas, la coyuntura social y política y sobre todo conocer algunas experiencias latinoamericanas y europeas que buscan, como nosotros, otros saberes y conocimientos emancipatorios nos daba una disponibilidad que nos permitió problematizar nuestra propia práctica investigativa y replantear el camino del trabajo para asumir un compromiso que mucho tenía de afectivo. Una afectación que nos dejaba tocados, que ya no nos permitía la inmunidad de la distancia, de estar a salvo en el lugar del que observa o del que cree saber.

\section{El compromiso con la situación}

Discutir estas tensiones con la propia práctica, confrontarlas con la experiencia cotidiana y la responsabilidad asumida con el colectivo, me llevó a la necesidad de aceptar un compromiso mayor que suponía asumir también sus dudas, contradicciones, titubeos y certezas como propias; en definitiva, de hacerlas comunes, hacerlas también mías. Esta situación despertó un dilema que se ampliaba en el campo del conocimiento científico y ponía en cuestión la subordinación que define y separa el saber del conocimiento y los vínculos de poder que se disputan en el campo epistémico (Albán, 2006; Botero, 2012; Escobar, 2003) y que en este caso demandaban una relación de horizontalidad y de reconocimiento mutuo.

La investigación que en la práctica se hace acción militante no puede ser entendida como una adición entre investigación y militancia, una sumatoria de dos lugares particulares que en un momento determinado pueden estar más o menos cercanos, solapados o incluso temporalmente fusionados, más bien refiere al modo de habitar la situación. Nos habla del compromiso, de un lugar difícil y casi siempre difuso que es investigar con los pies metidos en el barro. De esta forma se aleja y cuestiona la figura del investigador (académico) desapegado que se acerca a su objeto de análisis con el interés de vincularlo a sus tesis previamente planteadas. De la misma forma problematiza la figura del militante o del intelectual orgánico que pretende con su saber previo desideologizar las comunidades (Botero, 2012; Colectivo Situaciones, 2003).

La investigación y acción colectiva parte del reconocimiento y valor que poseen las propias prácticas y experiencias como verdaderas productoras de saberes y conocimientos situados y contextuados en su dimensión histórica y relacional. El método de investigación y acción reconfigura la relación tradicional de sujeto-objeto para transformarla en una relación sujeto-sujeto. La investigación militante se ubica en un lugar que se mueve entre la teoría y la práctica con la intención de coproducir conocimientos, saberes y modos de sociabilidad alternativa, a partir de la potencia que poseen los saberes subalternos (Colectivo Situaciones, 2003).

La investigación militante no trabaja a partir de un conjunto de saberes propios sobre el mundo, ni sobre cómo debieran ser las cosas. Muy por el contrario, la única y dificultosa condición del militante investigador es la de permanecer fiel a su "no saber". En este sentido, es una auténtica antipedagogía (como quería Joseph Jacotot) $^{2}$ (Colectivo Situaciones, 2003: 1-2).

En este sentido pensar en la idea de militante investigador abre más preguntas que respuestas. Es otro lugar que se mueve, que se resignifica en la experiencia inmediata, cotidiana y toma forma en el trayecto recorrido. Sin embargo, la posibilidad de que esto pueda ser pensado y tratado se produce en un momento concreto, cuando las resistencias toman formas nuevas y ya no se presentan únicamente bajo modos politizados, mucho menos partidistas, donde la dimensión de lo político le gana terreno a la política.

\section{El caso de la Cooperativa Integral Catalana}

La iniciativa de la CIC realiza un recorrido colectivo que podríamos ubicar entre el 2006 y 2008, cuyos antecedentes son algunas experiencias como la del infoespai o la cooperativa altercoms que funcionaron en aquel momento como nodos de difusión y comunicación de movimientos sociales. Durante estos años se fueron sedimentando una serie de experiencias, prácticas y encuentros que dieron lugar, en mayo de 2010, a la posibilidad de conformar una cooperativa. Esta forma jurídica permitía canalizar y dar soporte a distintas experiencias marginales 
de economía social y solidaria al tiempo que daba apoyo a otros proyectos que venían desarrollándose en distintos ámbitos.

El contexto de expansión y consolidación del proyecto está vinculado a los acontecimientos del $15 \mathrm{M}$ que provocaron el surgimiento de nuevos espacios y formas de participación y contestación ciudadana al tiempo que reforzaron y visibilizaron otras experiencias, algunas de ellas con larga trayectoria local. Sin duda las raíces históricas en Cataluña del cooperativismo, el anarquismo (con el lugar que ocuparon históricamente los ateneos libertarios), los movimientos vecinales o el movimiento okupa en los 90, fueron algunas de las experiencias que han influenciado y aún perviven, a veces sin ser demasiado conscientes, en las lógicas y modos de organización de espacios como estos.

Durante este proceso la CIC ha ido conformando un proyecto cuya característica principal, a primera vista, es su alta heterogeneidad y diversidad discursiva. Aparece así como enunciadora de un discurso vinculado al movimiento altermundista de resistencia global, a la soberanía alimentaria, al movimiento de software libre o las propuestas del pensamiento decrecentista que en plena efervescencia, durante los meses que siguieron a mayo del 2011, se mezclaron con otras demandas. Esta diversidad de experiencias e iniciativas, que confluyeron en este como en otros espacios, cuestionaron una vez más la forma de pensar lo político y confirmaron nuevos modos de politización que requerían, por tanto, nuevos esquemas interpretativos que nos ayudaran a pensar los acontecimientos.

Sin embargo en estos últimos años parecería que se han conquistado nuevas relaciones de fuerza que han producido planteamientos más o menos novedosos que pujan por un espacio dentro de la geometría política, sobre todo en el sur de Europa (concretamente en España y Grecia), que mira con mucho interés los procesos latinoamericanos de las últimas décadas. Tal vez uno de los riesgos o interrogantes que puede plantearse en este escenario es que la repolitización actual de estos espacios como forma de construir una hegemonía, de dar contenido a ese significante vacío del que habla Laclau (2010), ponga nuevas resistencias a la posibilidad de plantear las variadas formas que toma la micropolítica al interior de los lugares donde trabajamos. Pensar en esta micropolítica nos plantea un reto, pero también una necesidad para pensar (nos) desde nuestras prácticas concretas sabiendo de la dificultad que supone moverse en un terreno tan escurridizo e incierto.

$\mathrm{Si}$ bien las intervenciones de la CIC han ido cobrando diferentes formas, afianzando argumentos y saberes en distintos ámbitos, brindando apoyo a proyectos de salud, educación, autogestión, creando red de proconsumidores y participando en diversos debates, una de sus especificidades ha sido la de crear una estructura que permitiera desarrollar actividades de forma autogestionada limitando los condicionamientos e intervenciones del Estado y las lógicas capitalistas. De esta forma se plantea como un movimiento en transición dirigido a construir un espacio (espacio-tiempo) autónomo donde las personas puedan organizar sus vidas más allá de los gobiernos y el capital construyendo lugares alternativos que se proyectan hacia la producción de bienes comunes.

La CIC se estructura en forma de red descentralizada a lo largo del territorio para enlazar y dar soporte a distintos proyectos autónomos y autogestivos en diversos ámbitos. Se organiza a partir de asambleas abiertas quincenales donde se toman las decisiones referentes a su funcionamiento y futuras acciones. Consta de seis nodos articulados con un promedio de cinco comisiones de trabajo (jurídica, educación, salud, vivienda, red de distribución de alimentos, tecnología, comunicación, proyectos productivos, de intercambio y moneda social, etc.). De esta forma la CIC propone la construcción de un sistema público cooperativo que se construye desde las bases del común y hacia el común compuesto concretamente por las comisiones de salud, educación, alimentación, transporte, vivienda y tecnología.

\section{Salud. La historia de un lugar}

Uno de los proyectos de la CIC es Aurea Social, este lugar está ubicado en calle Cerdeña, a unos 100 metros de la Sagrada Familia, es una ubicación y un espacio privilegiado dentro de la ciudad de Barcelona. Originalmente el lugar fue una empresa familiar dedicada a las terapias alternativas. En 2010, la empresa abre sus puestas como clínica de salud con un edificio de $1400 \mathrm{~m}^{2}$ completamente equipado. Cuenta con 10 salas de consulta, una de ellas acondicionada con equipamiento odontológico, tres recepciones (dos en la planta baja y una en la primera planta), una centralita, lavadero y cocina completamente equipada, 
varias salas de reuniones, un espacio para eventos y conferencias con equipo audiovisual, vestuarios, ascensor y terraza.

La apertura de la empresa familiar se produce en un momento complicado, cuando la crisis ya era acuciante y en pocos meses el proyecto es abandonado tras asumir una deuda bancaria desorbitada a la que no puede hacer frente. Ante esta situación los socios se ponen en contacto con la Cooperativa Integral Catalana (CIC) y deciden entregarles las llaves para que el esfuerzo puesto en el proyecto familiar no caiga en el vacío y así evitar que el banco tenga el espacio y el proyecto inactivo. A partir de aquí la cooperativa llega a un acuerdo con la familia a la que le ofrece asesoría jurídica y asume la negociación de la deuda con la entidad bancaria. El traspaso de la empresa a la CIC es progresivo, mientras tanto se va gestando el proyecto Aurea Social promovido por la misma cooperativa. Finalmente se decide hacer uso del lugar como "sede" física de la CIC y concretar el nuevo proyecto Aurea Social que ya tenía cierto rodaje sobre todo en lo referente a iniciativas de salud y educación autogestionada.

Es así como en los primeros meses de 2012 Aurea Social se constituye como nuevo proyecto autónomo cooperativizado de la CIC. De alguna manera se conforma como una extensión de los ámbitos de acción de la cooperativa con una clara continuidad en las bases ideológicas y organizativas. El proyecto supone la colectivización del edificio para uso común de iniciativas y actividades afines con el ideario de la cooperativa.

Inmediatamente se comienza con una campaña de colectivización que tiene dos ejes fundamentales: informar y concienciar sobre el valor y posibilidades que supone contar con un espacio común con esas características, libre y abierto a toda la comunidad, y promover la compra común del inmueble para evitar que se lo quede el banco y así garantizar el desarrollo de un proyecto de carácter social y asambleario.

Durante 2012-2013 la comisión de salud de la CIC, ubicada físicamente en Aurea Social, fue una de las iniciativas más visibles de la cooperativa debido a la trayectoria anterior y porque era un espacio abierto a la comunidad con un alto poder de convocatoria. Durante ese año pasaron muchos profesionales, médicos/as, enfermeros/as, trabajadores/as sociales, personas con dolencias que la medicina oficial no había sabido dar respuesta y sobre todo terapeutas de las más variadas escuelas y perspectivas. En este espacio se cruzaron durante meses, colectivos que intentaban conformar una plataforma por la defensa de la salud pública, profesionales que trabajaban con colectivos "vulnerables", psicólogos y algunas otras propuestas que en su conjunto resultaba muy difícil definir, donde dominaban discursos heterogéneos, ambiguos a veces contradictorios.

A lo largo de ese año se articuló una red territorial compuesta por distintas iniciativas vinculadas al ámbito de la salud conformada por núcleos y nodos, algunos de los cuales ya estaban activos y se comenzó a trabajar sobre la posibilidad de crear un Sistema Mutualista Mancomunado en Salud.

El planteamiento del colectivo era bastante ambicioso y necesitó replantearse varias veces, entre otras cosas debido a la alta movilidad de las personas que colaboraban y por la diversidad de miradas y perspectivas que allí se hacían presentes.

\section{Errando con la salud}

A comienzos de 2014 la oficina de salud se encontraba en un impasse, así que la cooperativa decide en asamblea permanente reabrir este espacio, ya que el proyecto, como se había pensado, no podía imaginarse sin alguna de sus comisiones básicas como era la de salud. A partir de esta decisión se conformó un grupo de personas interesadas, algunas de ellas ya habían estado vinculadas a la etapa anterior, y otras fueron invitadas por la comisión de coordinación de la CIC como fue mi caso.

El grupo que trabajó durante todo ese año estuvo formado por una estudiante de medicina, una persona que participaba en un grupo de apoyo mutuo para gente con experiencias de internamiento psiquiátrico (GAM), cuatro personas cuyos saberes provenían de la medicina china y de otras terapias (llamadas) alternativas y yo.

En este escenario es donde mi compromiso con el colectivo toma todo su peso. El proceso de colaboración, que en un primer momento era más bien de tipo organizativo en algunas cuestiones relacionadas con la gestión del espacio de Aurea Social, pronto se convirtió en una implicación directa. Es en esos momentos cuando siento que la situación me exige repensar el aspecto metodológico de la investigación.

Ese estar implicada en los problemas, en las contradicciones, ambigüedades y certezas del colectivo se hacía cada vez más presente y las preguntas 
iniciales de la investigación comenzaban a desplazarse ante la urgencia de las demandas cotidianas del colectivo que muchas veces estaban ligadas a aspectos más bien operativos.

Durante y después del trabajo etnográfico que me había llevado hasta allí intentaba saber cómo podía continuar con la investigación y al mismo tiempo resolver algunos de los dilemas éticos que problematizaban la práctica investigativa. Era evidente que a nadie en la cooperativa le interesaba demasiado en qué iba mi trabajo de investigación, sino el vínculo que íbamos construyendo cada día con la convivencia y colaboración en distintos asuntos de la gestión del lugar o en las eternas jornadas asamblearias que se hacían una vez por mes durante tres días y que eran verdaderos espacios de discusión y formación.

Cada vez más sentía que la investigación tenía sentido en la medida que también lo tuviera para el colectivo. El tema no era solo devolver los resultados, en caso de que los hubiera, sino que ese estar allí cobrara sentido, se hiciera significativo, o al menos útil para el grupo. Ese estar allí, en un tiempo y un espacio concreto, con toda la carga de dudas y contradicciones pero también con las potencias que ahí se desplegaban, era cada vez más ese habitar la situación.

¿Cuál fue el trabajo de esos meses? Nos dedicamos a la recopilación y lectura detenida de los materiales producidos en la etapa anterior. Recuperación de contactos, reactivación de direcciones y listas de correos electrónicos, y revisión de las actuaciones y formaciones que se habían hecho en el ámbito de la salud.

Después de largas y agotadoras jornadas de trabajo acordamos seguir la línea de trabajo que se había desarrollado anteriormente, pero esta vez partiríamos de la problematización del concepto de salud. Cuestionar las relaciones de poder definidas por la figura del terapeuta y el paciente reforzando la idea de autogestión de la salud desde una perspectiva mucho más amplia que la biomédica y la posibilidad de pensar en otras terapias no reconocidas por el sistema público sanitario estatal, recuperando otros saberes, conocimientos y prácticas en salud. (Esto último estuvo fuertemente marcado por la propia historia del espacio, ya que como mencioné más atrás el edificio había sido un centro de terapias alternativas y esto era una presencia que se manifestaba de muy variadas formas, a veces con mayor o menor sutileza).
Trabajamos cuestionando aquellas acciones en salud asentadas en formas individuales e individualizantes para pensar las afecciones particulares siempre imbricadas en lo social y comunitario sin perder de vista la situación y el contexto que las envuelve.

Pero sobre todo queríamos escapar de la falsa dicotomía salud/enfermedad y también de la idea de la prevención vinculada siempre a un estado potencial de enfermedad; queríamos pensar las dolencias, nuestras afecciones de otra forma, sin caer en psicologismos, incluso pensarlas como posibles lugares de resistencia, como manifestaciones encarnadas del malestar que nos provoca esta falsa categorización de la vida y la existencia. En esta construcción de esquemas arbitrarios a los que nos arrojan y nos dicen quiénes somos y por tanto qué lugares ocupamos. Una exterioridad que nos nombra, nos clasifica, nos expulsa y otras nos identifica arbitrariamente. Y para esto necesitábamos formarnos, comprender las transformaciones del sistema-mundo así que emprendimos un período de autoformación, primero abocadas al ámbito de la salud porque allí estaban los intereses del grupo. Organizamos talleres, actividades y una formación en psicología social y comunitaria con la intención de problematizar aquellas perspectivas individualizantes que nos llegaban bajo distintas formas y discursos, a veces fácilmente detectables, pero otras no tan evidentes.

Este no era un camino fácil con el que pudiéramos avanzar sobre todo con planteamientos tan distintos, algunos no lo veían tan claro y creían más urgente dar soporte a otras situaciones injustas que estaban en la agenda política del momento, otros estaban más interesados en concretar acciones directas en salud y así dar contenido a la propuesta de la CIC. Hay que decir que el contexto sociopolítico de estos años en relación con la manera de entender la salud y los servicios que ofrece el sistema de sanidad público estatal ha estado brutalmente marcado por las políticas neoliberales y sus lógicas privatizadoras y mercantilizantes que parecen atravesarlo todo, incluso las propuestas, discursos y demandas que llegaban a la cooperativa y que se decían críticas.

Mientras tanto fuimos redactando un texto base con los principios, objetivos y el encaje que creíamos podía tener el proyecto dentro de la estructura de la CIC. Reactivar y dinamizar la compleja red de proyectos, nodos y núcleos vinculados a la salud que proponía la CIC no era tarea fácil y pensar en 
poner en marcha un sistema mutualista mancomunado parecía demasiado ambicioso para los recursos con los que contábamos.

Después de unos meses la urgencia de los distintos asuntos que abordamos hizo que nos olvidáramos en la práctica de las sutiles fuerzas que tejen los vínculos y nos encontramos desconcertadas en medio de situaciones a las que no sabíamos cómo habíamos llegado y que producían ruidos a nuestro proceso. Una vez más, como otros colectivos, confundimos lo urgente con lo importante.

\section{Memoria del presente}

Criticar los errores y destiempos repetidos por nosotros y otros colectivos era, sin duda, lo más fácil pero también nos confrontaba con nuestras propias prácticas. El reto estaba en ir más allá y detectar qué cosas sí valían la pena de nuestra experiencia y qué pasaba en estos tiempos y destiempos que habitábamos y nos habitaban.

En este recorrido nos propusimos ir registrando los acontecimientos, en la medida de lo posible, para reconocer el trayecto, una errancia que nos permitiera cierta perspectiva y a la vez un aprendizaje del proceso que nos ayudara a ir construyendo nuestra propia memoria, que nos permitiera recorrer un camino posible de acciones trazado de preguntas significativas, una suerte de gramática compuesta de preguntas.

Creíamos, y todavía lo hacemos, que por medio del vínculo que construíamos en el trabajo cotidiano podíamos pensar cosas que sin este encuentro no serían posibles. Que el trabajo colectivo y colaborativo son parte de la materia pensante desde la que uno (se) piensa y problematiza la existencia. Por tanto se trataba de propiciar un espacio donde construir colectivamente un pensamiento otro, emancipatorio donde no importaba mucho si hablábamos de salud, educación, soberanía alimentaria o tecnológica.

\section{Experimento, gestión y cansancio}

Después de un año el cansancio vuelve con fuerzas renovadas. Se suma a otros cansancios y a otros de nosotros. No nos toma por sorpresa, el trayecto estuvo plagado de señales, de pequeños cansancios.

Hacer, sostener las experiencias y responder a las demandas del colectivo, demandas ya definidas de antemano, proyectos previos a los que teníamos que dar contenido y mantener las propuestas, muchas veces con el propio cuerpo, comenzó a hacerse cada vez más palpable y ya no pudimos mirar para otro lado. Caímos en la trampa. Esas urgencias a la que nos abocamos respondían a las demandas de la organización y a veces parecían estar desconectadas de las personas que conformábamos el proyecto de la CIC. Teníamos que dar contenido a una estructura que a veces parecía estar vacía.

Nos tomamos unas vacaciones esperando volver con fuerzas renovadas, buscamos ilusionarnos otra vez. Algunos se fueron, otros se incorporaron. Durante algo más de un mes trabajamos sobre un plan estratégico que nos marcaría el rumbo y planteamos la urdimbre sobre la que tejeríamos nuestras acciones, la formación e investigación, la red de colectivos e iniciativas con las que queríamos trabajar; pensamos cada uno de estos ejes como una unidad, ejes o líneas que no podían pensarse separadamente.

Pero el cansancio ya estaba ahí y se manifestó como otras veces y casi con las mismas formas. Teníamos que asumir que plantear el cierre de la oficina de salud era necesario, no sabíamos si definitivo pero había que entenderlo como un acto de responsabilidad frente al colectivo y de respeto a nuestro propio proceso. Esta decisión nos daba miedo ¿teníamos que reconocer un nuevo fracaso de la oficina de salud o debíamos pensar que la necesidad de un cierre no es más que una parte de ese proceso? Pero sobre todo nos invadía una profunda tristeza. El agotamiento nos pedía parar, abrir el pensamiento, poner en práctica una política del cuidado como la habíamos pensado para cualquier otro proceso de lo que llamábamos salud-vida, pero sobre todo nos hablaba del mundo, de nosotros. ¿Qué nos pasó? ¿Cómo enraizaba nuestra experiencia en las prácticas y acciones de la CIC? ¿Qué cosas sí habían valido la pena y qué aprendimos de aquello, si es que habíamos aprendido algo? Era una especie de exceso de verdad que nos exigía ordenar las preguntas, sedimentarlas, antes de lanzarnos a buscar otras para iniciar un camino nuevo.

\section{Conclusión Primera persona}

Retomar la primera persona en este texto me resitúa nuevamente y me obliga a revisar el trayecto. Había llegado a la CIC con unas preguntas que lejos de quedar sin respuesta se resignificaron y ampliaron, añadiendo matices y nuevos interrogantes. 
La cuestión ética planteada en la primera etapa de la investigación, en relación con el acceso al campo, estuvo presente en todo el recorrido pues problematizaba el lugar que elige ocupar el investigador/a con relación a la realidad que desea estudiar. No se trataba de volver sobre la ya manida relación entre sujeto-objeto y la imposible pretensión de objetividad, sino de trabajar sobre la situación, una opción que es ante todo "política de la localización", que nos habla del territorio concreto desde el que hablamos, pensamos, actuamos e investigamos y por tanto desde el que producimos saberes y conocimientos contextuados que se validan en la propia práctica y la experiencia compartida.

Las características del espacio, los vínculos construidos en todos esos meses de trabajo, las afinidades y disidencias encontradas demandaban una implicación, un compromiso con el colectivo que exigió un replanteo metodológico.

Este cambio no fue fácil, pues supuso también poner en suspenso las preguntas iniciales que me había planteado en la investigación para asumir las que demandaba el colectivo, aunque a veces parecían llevarme muy lejos del tema que me había propuesto investigar, muchas de las preguntas iniciales de alguna forma fueron encontrando respuestas, ya que es en el hacer cotidiano donde se despliegan saberes, conocimientos y disponibilidades que toman sentido y significado en ese hacer implicado, en la espontaneidad, en la simultaneidad y en las contradicciones que nos interpelan.

Aceptar la necesidad de un cambio metodológico era reconocer el lugar de igualdad en un "no saber" provocador y abismal que se encontraba con las formas de experimentación y resistencia del propio colectivo. Un hacer que no pretende liberar o concienciar, sino que se produce en una relación de horizontalidad que se piensa emancipadora en sí misma (y que por eso pide coherencia también en el campo metodológico). No parte de una igualdad abstracta y universal a la que hay que llegar en un momento determinado, sino de una igualdad que es punto de partida en los vínculos que establecemos y que, sin embargo, no contradice el sabernos diversos. Por esto, y a pesar de esto, las preguntas se tornan siempre preguntas educativas, enredadas en sus implicaciones éticas y epistémicas.

\section{Agradecimientos}

A mis compañeras y compañeros de la CIC que me acompañan en este caminar preguntando que a veces se hace errancia, pérdida y encuentro. $\mathrm{Al}$ apoyo y la confianza del Grupo INTER y a la lectura atenta de Patricia y Santiago.

\section{Referencias Citadas}

Albán, A.

2012 Epistemes “otras”: ¿Epistemes Disruptivas? Del Atlántico Sur, K. A. Revista de Antropología y Ciencias Sociales 6: 22-34, Buenos Aires, Argentina.

Escobar, A.

2003 Mundos y Conocimientos de otro modo. El Programa de Investigación de Modernidad/Colonialidad Latinoamericano. Tabula Rasa 1: 51-86

Botero, P.

2012 Investigación y acción Colectiva-IAC-una Experiencia de Investigación Militante. Utopía y Praxis Latinoamericana: revista internacional de filosofía iberoamericana y teoría social 57: 31-48.

Colectivo Sin Ticket

2004 Errancias. En Nociones Comunes. Experiencias y Ensayos entre la Investigación y Militancia, editado por Colectivo Sin Ticket, 167-190. Traficantes de sueños, Madrid, España.

Colectivo Situaciones

2003 Sobre el militante investigador. Buenos Aires. http://eipcp.net/transversal/0406/colectivosituaciones/es/print (2 abril 2013)
Hardt, M.; Negri, A. 2002 Imperio. Paidós, Barcelona.

Laclau, E.

2010 La Razón Populista. Fondo de cultura Económica.

Muñoz Tamayo, V.

2006 Condiciones "post y asociatividad juvenil: preguntas por lo político. En México y Chile. última Década 25. Valparaíso: CIDPA.

Pessoa, F.

2008 Espólio Fernando Pessoa. Archivo de cultura portuguesa contemporánea. Biblioteca Nacional de Portugal. purl. $\mathrm{pt} / 1000 / 1 /$

Ranciére, J.

2002 El Maestro Ignorante. Cinco lecciones sobre la emancipación intelectual. Laertes, Barcelona.

Vercauteren, D.; Müller, T.; Crabbé O.

2010 Micropolíticas de los Grupos. Para una ecología de las prácticas colectivas. Traficantes de sueños, Madrid, España.

Virno, $\mathrm{P}$.

2003 Multitud y clase obrera. Entrevista. Contrapoder.

Zibechi, R.

2007 Dispersar el poder. Virus, Barcelona. 


\section{Notas}

$1 \quad 15 \mathrm{M}$ es el movimiento ciudadano formado a partir de las movilizaciones del 15 de mayo del 2011 en diversas plazas de España, fundamentalmente en Plaza del Sol de Madrid. Acontecimiento que aglutinó el descontento social de amplios sectores de la población que reclamaban una democracia más participativa en oposición al bipartidismo (PP-PSOE) y al dominio de la banca y las corporaciones. La experiencia del 15M, la llamada Primavera Árabe junto con otros movimientos sociales emergentes a nivel global, han expresado nuevas formas de autoorganización política que desafían las lógicas gubernamentales para dar respuesta a las demandas de la ciudadanía.

2 A lo largo de la historia del maestro Jacocot, Ranciére escribe un texto provocador para la pedagogía pues sostiene que puede enseñarse sin saber, para ello despliega un tratado sobre la igualdad (de las inteligencias) y critica la relación pedagógica construida por el pensamiento moderno. El texto desmitifica el rol pedagógico que se creyó portador de igualdad y libertad al tiempo que confronta la figura del intelectual como reproductor de la desigualdad social que pretende combatir (Ranciére 2002). 
